\section{Cahiers franco-canadiens de l'Ouest}

\section{alias ROSSEL VIEN}

\section{J.R. Léveillé}

Volume 32, numéro 2, 2020

\section{L’énigme Rossel Vien}

URI : https://id.erudit.org/iderudit/1072137ar

DOI : https://doi.org/10.7202/1072137ar

Aller au sommaire du numéro

\section{Éditeur(s)}

Presses universitaires de Saint-Boniface (PUSB)

\section{ISSN}

0843-9559 (imprimé)

1916-7792 (numérique)

Découvrir la revue

\section{Citer cet article}

Léveillé, J. (2020). alias ROSSEL VIEN. Cahiers franco-canadiens de l'Ouest, 32(2), 313-335. https://doi.org/10.7202/1072137ar

\section{Résumé de l'article}

L'article explore la venue à l'écriture de l'auteur Russel Vien qui a publié sous nom de plume tout au cours de sa vie. Que cache et que révèle cette course fuyante vers l'anonymat qui a produit un auteur salué par la critique, connu en histoire et méconnu en fiction? Les différentes sections de l'article analysent chaque pseudonyme, ainsi que la réception critique initiale d'une oeuvre « inconnue » où nous découvrons les enjeux de la personnalité mystérieuse d'un pionnier de l'écriture homosexuelle et d'une modernité formelle.
Tous droits réservés (c) Centre d'études franco-canadiennes de l'Ouest (CEFCO) et Presses universitaires de Saint-Boniface (PUSB), 2020
Ce document est protégé par la loi sur le droit d'auteur. L'utilisation des services d'Érudit (y compris la reproduction) est assujettie à sa politique d'utilisation que vous pouvez consulter en ligne.

https://apropos.erudit.org/fr/usagers/politique-dutilisation/ 


\title{
alias ROSSEL VIEN
}

J.R. LÉVEILLÉ

\begin{abstract}
RÉSUMÉ
L'article explore la venue à l'écriture de l'auteur Russel Vien qui a publié sous nom de plume tout au cours de sa vie. Que cache et que révèle cette course fuyante vers l'anonymat qui a produit un auteur salué par la critique, connu en histoire et méconnu en fiction? Les différentes sections de l'article analysent chaque pseudonyme, ainsi que la réception critique initiale d'une oeuvre «inconnue» où nous découvrons les enjeux de la personnalité mystérieuse d'un pionnier de l'écriture homosexuelle et d'une modernité formelle.
\end{abstract}

L'auteur est né Russel Vien en 1929, tel qu'attesté par le certificat de naissance. Ce n'est qu'en 1955 à la parution de son Histoire de Roberval, cour du Lac-Saint-Jean, 1855-1955 qu'il signe Rossel Vien. La transformation est légère, mais on peut dire que c'est déjà un pseudonyme (il s'affiche comme étant l'auteur Rossel Vien) qui deviendra celui officieux de son état civil. C'est le premier d'autres noms de plumes qui suivront ${ }^{1}$.

Cette prise en main d'individuation n'est pas sans rappeler le célèbre slogan d'un dénommé Jean-Nicolas-Arthur Rimbaud : «JE est un autre». C'est bien de cela qu'il s'agit. Une lettre de l'auteur en herbe, adressée à l'abbé Victor Tremblay, au moment (1947) où il songe à écrire «une monographie de Roberval» est signée «Russell [sic] Vien ${ }^{2}$. C'est la première étape conduisant au nom Rossel Vien. Cette dénomination relève aussi de ce que j'ai pu qualifier de «combat spirituel» (Léveillé, 2020, p. 9). Dans un document qu'il a rédigé à l'intention de Raymond Hébert, Bernard Vien, le frère de Rossel, affirme : «Il semble assez clair que Russell [sic] n'aimait pas son nom surtout 
à partir de l'Histoire de Roberval. En quittant les clercs St-Viateur, il a senti et vécu une délivrance, et une grande liberté? Il m’a dit qu'il a perdu dix ans de sa vie ${ }^{3}$.»

Le nom d'origine demeure attesté jusqu'à la fin de sa vie. En 1947, Russel Vien, alors au Séminaire de Joliette, obtient une «Mention» dans la catégorie Littérature au $X^{e}$ Concours de Vacances de l'Action catholique de la jeunesse canadienne française (Anonyme, 1947) ${ }^{4}$. Une photo de classe au Séminaire de Joliette en 1951 l'identifie comme Russel Vien, son certificat de baccalauréat l'année suivante est attribué à Russel Vien $(\mathrm{ABV})$.

Dans les archives de Bernard Vien, un passeport canadien pour les années 1973-1978 émis au nom de Rossel Vien (page 2) comporte un renvoi (page 4) qui indique que le nom sur le certificat de naissance est Joseph Paul Émile Russel Vien ${ }^{5}$.

Le certificat de statut indien qu'il obtient en 1985, l'identifie ainsi : VIEN, J. Paul Émile Russel - Mont. Lac St-Jean 3598 (ABV), statut dont l'auteur n'était pas peu fier puisqu'il écrit dans son récit Retour à Saskatoon: «[...] j'ai ma carte, maintenant, avec le nom de la tribu [...] ma carte d'identité, numérotée. Je suis Déné, hourrah!» (Vien, 1990) ${ }^{6}$.

Même en 1987, Benoit Beaulieu dans sa recension de Et fuir encore, signé Gilles Delaunière (autre pseudonyme de RV), pour le Dictionnaire des cuvres littéraires du Québec (Beaulieu, 1987, p. 321-322) reconnaît Delaunière comme étant toujours Russel Vien. Aurélien Boivin, responsable de la section «Roman, conte et nouvelle» du dictionnaire avait été le premier, l'année précédente, à identifier Gilles Delaunière comme étant Rossel Vien dans Littérature du Saguenay-Lac-Saint-Jean : répertoire des cuvres et des auteurs (Boivin \& Bourgeois, 1986, p. 198). Dans la première édition du répertoire (1980), le lien avec Delaunière n'avait pas encore été fait, mais Boivin avait donné le nom de naissance (Russel) de l'auteur de l'Histoire de Roberval (Boivin \& Bourgeois, 1986, p. 145).

Enfin, le rapport d'autopsie du bureau du médecin légiste, le 4 mai 1992, identifie l'auteur comme VIEN, Russell [sic] $]^{7}$

RV publiera, au cours des ans, plusieurs œuvres sous le nom de Rossel Vien, et on peut remarquer, à une ou deux 
exceptions près ${ }^{8}$, que ce nom est réservé aux ouvrages de nature historique et à son travail de journaliste. Voyage sans suite publié en 1962 dans les Écrits du Canada français peut être considéré de même nature puisqu'il s'agit d'un carnet de voyage rassemblant les annotations que fit RV lors d'un séjour en Europe et en Afrique en 1958. Récit qui fut vertement critiqué par Jean-Éthier Blais :

$\mathrm{Du}$ «Voyage sans suite» de M. Rossel Vien, il n'y a strictement rien à dire. Ce jeune auteur a rapporté de ses pérégrinations, sac au dos, des souvenirs qui indifférent. C'est qu'au fond, il ne nous raconte rien. [...] Pas une anecdote saillante, pas un bon mot, rien qui nous apprenne quoi que ce soit. [...] C'est à vous dégoûter des voyages. (Blais, 1964, p. 15)

\section{Gilles Delaunière}

Tout comme Rossel Vien a fait une entrée remarquée en littérature historique ${ }^{9}$, il apparaît avec éclat dans le domaine de la fiction en publiant en 1960, sous le pseudonyme de Gilles Delaunière, «Un homme de trente ans» aux Écrits du Canada français. Le récit attire l'attention de critiques bien connues, tel Gilles Marcotte :

Le récit mêlé de réflexions que nous livre Gilles Delaunière sous le titre «Un homme de trente ans» est bien la confession la plus nue, la plus directe, qu'il m'ait été donné de lire au Canada français. Elle est si directe, à vrai dire, qu'on ressent d'abord une certaine gêne. D'autant que le thème principal : l'amour socratique, en est évidemment assez scabreux. Mais je m'empresse d'ajouter qu'il n'y a rien de bas dans cette confession, et qu'au contraire un certain ton, dès l'abord, nous avertit que nous avons affaire à toute autre chose qu'à un grossier déshabillage. [...] Quelque part, ailleurs, il y a «la vraie vie», et le désespoir n'est pas si total qu'on ne jette un regard de temps à autre vers une petite étoile vacillante. Rien n'est perdu, de ce qui s'exprime avec une aussi brûlante, une aussi humble sincérité. Et à cause précisément de cette sincérité, de cette vérité, le récit de Gilles Delaunière dépasse l'intérêt du simple document psychologique; il atteint à la qualité d'une véritable communication. Notre littérature gagne un écrivain, et «Un homme de trente ans» ne devrait être qu'un début (Marcotte, 1960, p.11). 
La recension la plus dithyrambique, signée G.G. ${ }^{10}$, paraît dans Le Nouvelliste de Trois-Rivières :

Gilles Delaunière (un pseudonyme) nous donne avec Un homme de trente ans, ce qui est peut-être le premier-né des chefs-d'oeuvre de notre littérature. [...] Une œuvre capitale, un document et je termine, encore sous le coup de l'émotion que m'a donné la lecture de ce récit simple et humain comme peuvent nous en donner les excellents écrivains. (G.G., 1960, p. 14)

On prévoit d'ailleurs de grandes choses pour l'auteur. On lit dans un entrefilet de La Presse : «Gilles Delaunière, dont le récit 'Un homme de trente ans' avait été très remarqué dans le Tome VI des Écrits du Canada français, aurait reçu plusieurs offres d'éditeurs français pour un prochain roman» (Anonyme, 1960, p. 28). Dans Le Devoir :

Parmi les textes les plus remarqués, il y a eu celui de Gilles Delaunière, un jeune écrivain qui tient à garder l'incognito, car ce nom est un pseudonyme. Trois éditeurs lui ont demandé un roman. Dans le prochain numéro [1961], il publiera une longue nouvelle intitulée : 'L'auberge des Trois-Lacs'. (G.H., 1961, p. 10)

En tout, Gilles Delaunière publiera cinq nouvelles dans les Écrits : les deux susmentionnées, Les aveugles de Matamoros en 1970, La coulée et Les roses de papier en 1981.

Gilles Marcotte pouvait connaître la véritable identité de l'auteur, car il figurait au comité de rédaction ${ }^{11}$ de la revue ${ }^{12}$. Tous savent toutefois, sans connaître l'identité de l'auteur, que Delaunière est un pseudonyme, car à la publication de sa nouvelle, la revue juge bon de préfacer le texte par cet avis :

Sous ce pseudonyme se cache un nouvel auteur canadien qui, pour des raisons bien précises, tient absolument à ne pas révéler sa véritable identité. Les lecteurs qui prendront connaissance de son essai de confession : Un homme de trente ans, comprendront pourquoi. Il s'agit d'une histoire vraie et vécue. (Écrits du Canada français, 1960, p. 156)

Réginald Martel le déplorera douze ans plus tard lors de la parution du recueil de nouvelles Et fuir encore de «Gilles Delaunière, à qui il faudrait dire qu'il a peut-être eu tort de masquer un nom connu d'un pseudonyme, parce que ses nouvelles, réunies sous le titre Et fuir encore, sont parmi les 
meilleures qui s'écrivent ici» (Martel, 1972, p. C3). Il n'est pas clair que Martel estime que RV a «eu tort de masquer...», car il connaît le nom véritable de l'auteur, ou car il estime que la personne bénéficierait d'être connue?

Il n'est pas impossible que dans ce tout de même petit milieu littéraire (quoiqu'en ébullition) à l'époque, l'identité de l'auteur fut connue quoique poliment tue. L'administrateur des Écrits et l'éditeur de Et fuir encore en 1972, Claude Hurtubise, le connaissait bien ${ }^{13}$. RV a d'ailleurs maintenu une amitié et une correspondance avec son éditeur qui lui écrivait de longues lettres sur l'état du milieu littéraire au Québec.

Le livre est plutôt bien reçu. Martel, en plus de souligner que les nouvelles de Delaunière «sont parmi les meilleures qui s'écrivent», vante la qualité de l'écriture : «le livre est aussi et surtout une leçon d'écriture et cela seul, si quelque chose compte, compte». Plus loin dans sa recension :

Celui qui signe Gilles Delaunière se soucie peu d'épater et, plutôt que de faire mentir le mensonge en calquant son écriture sur celle des autres, il choisit comme naturellement le style qui convient à son entreprise d'exorciste. Parce qu'elle est périlleuse, cette tendance vers la simplicité paraît être la bonne. Le vécu est un fonds inépuisable auquel Gilles Delaunière pourrait, devrait revenir, pour écrire encore, et mieux écrire si possible, sa belle symphonie concertante.

Roger Duhamel dans Le Droit souligne la naissance d'un auteur : «[U]n écrivain authentique, déjà sûr de ses moyens [...] Il possède un sens très juste de la poésie, de cette poésie qui, telle l'élégance, atteint à son plus haut période dans la mesure où elle ne s'étale pas, mais irrigue en un courant souterrain l'imagination de l'écrivain.» (Duhamel, 1972, p. 13)

Il qualifie les textes qu'il recense d' «œuvres d'une facture remarquable», il évoque «des gammes et [...] des vocalises d'une justesse rare.»

Pour sa part, Jean-Éthier Blais, qui sait de quoi il retourne, rédige une recension mitigée. Il sent un talent, il évoque en sourdine la qualité du ton de l'écriture et du tableau qui est brossé, mais l'étouffement qu'il y ressent lui déplaît : 
M. Gilles Delaunière écrit des nouvelles où le vide est puni. Son héros, et tous ses personnages, ont une âme lourde de péchés. Ils aiment leur propre sexe et ne parviennent pas à accepter leur condition en marge de tout. Ce sont des fantômes que leur éducation, qu'elle soit catholique ou protestante, a marqué au fer rouge. Ils se plaignent, ils pleurent, s'analysent, se regardent sombrer lentement dans l'acceptation de la loi. Ils se fuient euxmêmes (Blais, 1972, p.14).

Le critique s'il peut apprécier certaines qualités d'écriture trouve le fondement vieillot : «La langue est souvent piétique, ce qui donne à penser que lorsque Gilles Delaunière aura laissé tomber le vieil homme et regardera vivre les autres en fonction d'eux-mêmes, il pourra écrire une oeuvre forte et pleine de feu. Tel quel, son livre ne lui rend pas justice. C'est dommage.»

Blais y décèle cette «atmosphère d'écartèlement» que notait douze ans auparavant Jean Paré dans Un homme de trente ans :

[L]'histoire pathétique d'un homme qui n'a pas choisi son sexe, qui a souffert de son éducation, de son complexe d'infériorité, de sa faiblesse. Son angoisse, sa solitude, son masochisme lui donnent l'horreur non seulement de la violence physique, mais de tout ce qui est physiologique. Il veut fuir, s'évader : il joue à la femme, au prêtre, au mystique. C'est l'impossible retour à de confortables entrailles. «Son âme s'est trompée de corps»; en de telles circonstances, 'le comble de la volupté est le martyre'» (Paré, 1960, p. 42).

Mon collègue Bernard Mulaire a raison d'estimer ${ }^{14}$ que Rossel Vien/Gilles Delaunière devrait figurer parmi les précurseurs de la littérature homosexuelle au Québec. Si, comme il le suggère, la clandestinité pouvait être souhaitable au début des années 1960, les années 1970 étaient plutôt celles de l'émergence, et RV de par son pseudonyme était quelqu'un qui s'affichait mais ne s'affirmait pas. «Ils se fuient eux-mêmes» dit Jean-Éthier Blais des personnages de Delaunière dans le recueil au titre si symbolique, Et fuir encore. "Il veut fuir, s'évader» affirme Jean Paré du narrateur d'Un homme de trente ans.

Tout le contraire d'une revendication assumée. Cette vieille dichotomie catho était déjà chose du passé, rejetée par les gays et les intellectuels de la Révolution tranquille : «le produit hypersensible d'une certaine société janséniste de chez nous, 
bigote et calculeuse, qui enseigne que l'homme nait méchant, que la chair est une punition du ciel et partant honteuse.» affirmait G.G. dans Le Nouvelliste.

Quoique les œuvres de fiction de RV sont généralement qualifiées de nouvelles, la plupart relèvent du «récit». Un homme de trente ans est d'ailleurs défini comme «une histoire vraie et vécue» dans l'Avis des Écrits du Canada français. En effet, la distanciation entre l'auteur et le narrateur est mince dans les fictions de RV et le «fictif», marginal, de sorte que la classification «nouvelle» apparaît comme un euphémisme pour «récit». Dans un document dans une chemise de correspondances variées sans date du Fonds Rossel Vien, RV explique sa conception de la nouvelle, précisant : "Ce genre permet beaucoup de franchise et d'intimité, sans que l'auteur ait à suivre les obligations formelles de la biographie, du reportage, ou de l'autobiographie.» (SHSB 0091/1445/5)

\section{Gilles Delanaudière}

Puis RV devient Gilles Delanaudière. Ce transfert de Delaunière à Delanaudière se fait à l'insu de l'auteur. L'erreur se présente dès 1972, puis se perpétue jusqu'aux années 2000. Dans son listage des parutions de l'année, Livres et auteurs québécois 1972 (Thério, 1972, p. 427) donne Gilles Delanaudière comme auteur de Et fuir encore. Il est probable que les chercheurs ayant consulté cette œuvre de référence pour préparer leurs propres publications aient répandu la coquille.

L'erreur est commise dès la parution du recueil. Victor Lévy-Beaulieu qui a pris le bouquin en estime écrit en mars 1972 : «Il y a ce beau mot dans Et fuir encore de Gilles Delanaudière, il y a ce qu'il appelle "les pays québécois" et que je m'approprie [...]» (Beaulieu, 1972a, p.14). Méprise qui sera répétée en fin d'année : «Il [Jacques Ferron] est même le premier écrivain d'ici à connaître aussi bien ces pays québécois dont parles Gilles Delanaudière dans Et fuir encore.» (Beaulieu, 1972b, p. 19)

En 1989, l'équipe de Réginald Hamel, John Hare et Paul Wyczynski identifiera, dans le Dictionnaire des auteurs de langue française en Amérique du Nord (Hamel, p.1336-1337), Gilles Delanaudière comme étant le pseudonyme de Rossel Vien. Qui plus est, le Dictionnaire le fait naître à Joliette (Roberval) en 1925 (1929). 
En 2001, Hamel corrige certaines données (lieu et année de naissance) dans son Dictionnaire des poètes d'ici, mais identifie toujours Vien comme Delanaudière, y ajoutant le pseudonyme (exact) de Gilles Valais, ainsi que des informations pertinentes (Guérin et Hamel, 2001, p. 1028). La réédition du dictionnaire (Guérin et Hamel, 2005, p. 1316) répète l'erreur. La bévue est maintenue lorsque Ismène Toussaint, sur son site, donne Gilles Delanaudière et Gilles Valais comme pseudonymes de Rossel Vien; elle est corrigée dans la version publiée dans l'Encyclopédie canadienne 2000 (Toussaint, 2000, p. 2582-2583).

\section{La fin de Gilles Delaunière}

Gilles Delaunière publiera deux autres nouvelles au Québec, dans les Écrits du Canada français, neuf ans après la parution de son recueil. Ce sont La coulée et Les roses de papier, toutes deux dans le $\mathrm{n}^{\circ} 43$ de la revue en 1981. Ce sera la fin de Gilles Delaunière.

Les relations deviennent tendues entre l'auteur et la maison d'édition Hurtubise $\mathrm{HMH}$ que dirige maintenant Marie José Thériault; aussi, le cheminement de ses manuscrits aux Écrits du Canada français prend un peu de lenteur. Son ami Claude Hurtubise, qui a quitté la maison d'édition qu'il a créée en 1960, se trouve toujours aux Écrits et laisse entendre à RV que la situation est difficile pour certains éditeurs ${ }^{15}$.

Février 1979, Hurtubise écrit ${ }^{16}$ à Rossel pour lui indiquer qu'il commence à lire les deux nouvelles que l'auteur lui a envoyées. Un an plus tard, la publication de ces textes n'est pas réglée. Hurtubise a en main la version remaniée de «Roses de papier» qui pourra paraître, dit-il, dans le $\mathrm{n}^{\circ} 44$, le $\mathrm{n}^{\circ} 43$ allant sous presse. Il a aussi reçu les rapports de lecture pour «Chibougamau» et «La coulée» qu'il achemine à RV. En fait, les «Roses de papier» ainsi que «La coulée» paraîtront toutes deux dans le $n^{\circ} 43$.

En août de la même année, RV s'adresse à Marie José Thériault, directrice littéraire chez $\mathrm{HMH}$ pour lui soumettre deux nouvelles en vue de composer un recueil, "Lettre de Maud» et «Les deux frères». Le 5 octobre, elle lui répond, refusant «Les deux frères», estimant que la «Lettre de Maud» «pourrait être sensationnelle» s'il acceptait certaines corrections, car, affirme-t-elle, son «français s'anglicise malheureusement». 
RV s'offusque devant ce qu'il considère une condescendance langagière, lui demande de lui rendre ses deux manuscrits et de résilier la clause de préférence dans son contrat avec $\mathrm{HMH}$, ce que la directrice fera, en lui indiquant toutefois qu'elle serait prête à recevoir d'autres textes de sa main. À la fin d'octobre, RV demande à Marie José Thériault de lui retourner aussi deux autres manuscrits («Le monastère» et «La statue») en sa possession. Il récidive une semaine plus tard, tout en reconnaissant qu'à la relecture il avait découvert des imprécisions dans «Lettre de Maud».

En septembre 1980, Claude Hurtubise écrit à RV lui demandant ce qui était advenu du recueil refusé par $\mathrm{HMH}$, et s'il s'était adressé, comme il le lui avait suggéré, à François Hébert aux Éditions Quinze. Deux mois plus tard RV proposera, en effet, un manuscrit aux Quinze, intitulé Le fils unique, qui comprend, outre la nouvelle éponyme, «Le monastère», «Lettre de Maud» et «La statue». L'éditeur reconnaîtra la qualité de l'écriture, mais y décèlera aussi des faiblesses de composition, et ne publiera pas.

RV n'abandonne pas pour autant. On trouve dans son Fonds une copie de lettre adressée à Victor-Lévy Beaulieu (qui avait été frappé par «les pays québécois» de Et fuir encore) dans l'intention de lui faire parvenir un manuscrit composé de «Le fils unique» et «Lettre de Maud». On ne sait si la lettre ou le manuscrit ont été postés.

Enfin, au tout début de janvier 1982, une lettre de Marie José Thériault accuse réception d'un autre envoi de l'auteur, sans doute celui qu'elle commente le mois suivant, indiquant que les corrections apportées à «Lettre de Maud» «n'ont pas suffi à faire de cette nouvelle la nouvelle sensationnelle qu'elle pourrait être», ajoutant que «Le fils unique» devrait être reconstruit.

Ultime tentative de publier au Québec, au printemps 1985, RV envoie une nouvelle, intitulée «Quantièmes» aux Écrits du Canada français. Le texte est donné en lecture. On n'en connaît pas le cheminement, sauf qu'en bout de ligne, il ne sera pas publié ${ }^{17}$.

Devant ces derniers échecs, on peut citer le post-scriptum de la lettre que RV adressait à François Hébert le 13 novembre 
1980 : «Gilles Delaunière est un pseudonyme que je n'aime pas tellement. Mais c'est celui que j'avais adopté en premier lieu.»

\section{Gilles Valais}

C'en est fini de Gilles Delaunière. Les nouvelles refusées, il ferme la porte au Québec et s'adresse dès lors à des éditeurs franco-manitobains. Presque tous les textes qui auraient paru au Québec sous le nom de Gilles Delaunière sont publiés au Manitoba sous le nom de Gilles Valais.

Il faut croire que RV aurait été prêt à vêtir les deux noms de plume, car au début des années 1980, pendant qu'il cherche toujours à publier chez les éditeurs québécois, il fait paraître Les deux frères (1982) aux Éditions des Plaines à Saint-Boniface, Manitoba, sous le nom de Gilles Valais ${ }^{18}$. Le recueil comprend la nouvelle éponyme, ainsi que «Chibougamau» et «Mathieu». Claude Hurtubise lui écrit en 1984 accusant réception de sa publication manitobaine; il lui fait savoir que les portes des Écrits lui demeurent ouvertes (sans succès, comme on l'a vu). À ce moment-là, le recueil Les deux søurs («Les deux sœurs» et «Lettre de Maud»), qui paraîtra en 1985, est en préparation de publication.

Ainsi, RV, l'auteur de nouvelles (Delaunière) qui est passé incognito au Québec, sauf de ses éditeurs et du petit monde à qui ils auraient confié le secret, publie tout aussi anonymement (Valais) au Manitoba. Tous les pseudonymes ne cachent pas un anonymat. C'était pourtant le cas de RV. Anonymat qui va se dissoudre petit à petit.

Au cours des années 1970, l'auteur Rossel Vien était connu au Manitoba pour ses essais historiques, plus particulièrement son histoire de La radio française dans l'Ouest et son travail autour de Louis Riel. Le public ne savait pas que RV était Gilles Delaunière (sauf possiblement, comme le suggère Bernard Mulaire $^{19}$, ceux qui avaient le pli de la soutane et leur élite laïque).

Lorsque Gilles Valais publie aux Éditions des Plaines en 1982, son identité n'est connue que de son éditrice, Annette SaintPierre, de gens de la maison d'édition, peut-être de quelques proches. 
En préparant le Répertoire littéraire de l'Ouest canadien, auquel RV collabore, Annette Saint-Pierre confie à Vien la rédaction de sa proprebio-bibliographie, qu'elle signera toutefois à sa demande ${ }^{20}$. Le Répertoire paraît en 1984 . On peut lire que Rossel Vien «[...] a publié des essais et des récits dans les Écrits $d u$ Canada français, des poèmes et, sous des pseudonymes, des recueils de nouvelles.» (Annette Saint-.Pierre, 1984, p. 355) Ainsi Rossel Vien se révèle lui-même, en 1984, comme ayant publié sous divers pseudonymes, sans préciser lesquels. Cherche-t-il à être découvert?

Il le sera. Comme nous l'avons vu précédemment, Aurélien Boivin en 1986 et Benoit Beaulieu en 1987 identifient Gilles Delaunière de son nom de naissance : Russel Vien.

Au cours de la deuxième moitié des années 1980, j'ai moi-même entrepris des recherches en vue de la publication de l'Anthologie de la poésie franco-manitobaine. Je me souviens d'avoir scruté les numéros des Écrits du Canada français à la recherche de poèmes de Rossel Vien ou de Gilles Delaunière, aussi, pour compléter la bibliographie de RV. Parue en 1990, 1'Anthologie identifiera clairement Rossel Vien avec ses pseudonymes (Gilles Delaunière et Gilles Valais) et établira une bibliographie de l'heure qui comprend la publication «à venir» du Fils unique de Gilles Valais (Léveillé, 1990, p. 355-363).

Rossel Vien qui venait de faire paraître sous le nom de Gilles Valais ${ }^{21}$ un deuxième recueil de nouvelles (Les deux sœurs, 1985) aux Éditions des Plaines a collaboré à la préparation de $l^{\prime}$ Anthologie. Il était très conscient du fait qu'il allait être identifié dans le volume. Il m'a lui-même fourni, en 1988, une série de poèmes (une douzaine, qui représente la majeure partie de sa modeste production dans le domaine ${ }^{22}$ ) dans laquelle j'ai choisi.

À la fin des années 1980, l'identité de Rossel Vien/Gilles Delaunière/Gilles Valais est bien établie. L'Anthologie de la poésie franco-manitobaine et Le fils unique de Gilles Valais paraissent la même année (1990). Mais le pseudonyme de Gilles Valais n'est plus un anonymat, c'est devenu un simple nom de plume, maintenu par l'auteur, connu de tous. D'ailleurs, lorsque le roman figure parmi les finalistes au Prix littéraire La Liberté dans le cadre des Prix littéraires du Manitoba en 1992, l'auteur Gilles 
Valais est appelé à lire avec les autres finalistes à la Bibliothèque publique de Saint-Boniface le 12 avril. C'est Rossel Vien qui se présente. Ce sera sa dernière prestation publique. Il est trouvé mort le $1^{\text {er }}$ mai 1992. Le rapport de l'autopsie identifie le défunt comme étant Russell Vien. Mort à peu près dans le nom avec lequel il est né23.

\section{Qui est Gilles?}

Voilà la question qui se pose. Delaunière disparaît pour Valais, mais Gilles demeure. Pourquoi cette prédilection pour le prénom des pseudonymes? Préférence marquée, car n'écrivaitil pas ci-haut à François Hébert que Gilles Delaunière était un pseudonyme qu'il n'aimait guère? C'est bien au Delaunière qu'il ne tenait pas puisqu'il conserve «Gilles» pour le remarier à Valais. L'état de la recherche autour de l'œuvre et de la personne de RV n'est qu'à ses débuts. On trouvera peut-être une réponse dans l'œuvre même de l'auteur, dans des passages déclencheurs comme celui de la nouvelle «Oriel» que souligne Jean-Éthier Blais : «Dès l'enfance, une rencontre le marque, celle d'un grand garçon qui, en rase campagne, l'embrasse» (Blais, 1972, p. 14). Ou dans un passage fondamental du récit «Un homme de trente ans»:

Ce qui devait naître était né depuis longtemps. À treize ans, j'avais aimé un de mes compagnons de classe, à l'école des Frères de Bellerive. Il avait de grands yeux noirs et des lèvres largement et admirablement dessinées. Il avait joué le rôle de la Sainte Vierge dans une saynète donnée dans la salle de l'école. Maquillé, sous un voile, son visage tendre était apparu plus tendre encore (Delaunière, 1960, p. 202).

Cette intuition m'est confirmée par des renseignements que j'ai pu obtenir des archives des Clercs de Saint-Viateur ${ }^{24}$ à Joliette. De 1940 à 1943, nous retrouvons dans la Confrérie des Saints-Anges, un dénommé Gilles ***, de quelques années plus jeune que RV. L'élève participe en 1941 et en 1942 à un spectacle, la Veillée de Noël. Les archives ne spécifient pas les rôles interprétés par les participants. Quoique ces données ne sont pas une preuve absolue, elles semblent, à l'heure actuelle, plutôt convaincantes.

Il vaut la peine d'ajouter toutefois que l'attitude de l'auteur à l'égard de ses pseudonymes devient plus insouciante, 
particulièrement à compter des années 1980. Il avoue son pseudonyme à François Hébert aux Quinzee $e^{25}$, en fait part à des tiers $^{26}$, publie dans l'Ouest, sous le nom de Gilles Valais ou de Rossel Vien, des textes préalablement destinées, sous le nom de Gilles Delaunière, à des éditeurs québécois ${ }^{27}$. L'anonymat requis à la publication $\mathrm{d}^{\prime}$ «Un homme de trente ans» en 1960, maintenu à la parution de Et fuir encore en 1972, se dissipe peu à peu. Le pseudonyme (le masque) cède au nom de plume (l'écriture). Les craintes de l'auteur et le caractère fuyant qui lui a été reproché par la critique au cours des premières années font place à une identité sexuelle de plus en plus assumée ${ }^{28}$. Comment lire autrement le titre de la nouvelle «Quantièmes», aussi révélatrice de l'être que fut le premier récit («Un homme de trente ans»)? La nouvelle, d'abord intitulée «Le désir», affiche en épigraphe une phrase de Georges Bataille. Le désir ne disparaît pas pour autant dans cette nouvelle, mais, enfin, il prend corps et âme. Le texte met en scène l'épigraphe de Bataille. Le désir n'est plus fugitif, c'est un enjeu qui se réalise, se concrétise et se dénombre dans les rencontres charnelles.

Malgré la modernité de l'écriture historique de Vien ${ }^{29}$, c'est plutôt dans la fiction de l'auteur qu'il faut chercher l'être, très précisément dans la forme de la nouvelle. La poésie «retient des éléments sublimés», le roman "comporte presque toujours des temps morts, des longueurs, des remplissages, des àcôtés» (SHSB 0091/1445/5) 30 $^{3}$ La nouvelle chez Vien est un récit de vie vraie. Il reconnaît que la «nouvelle permet des formes d'écriture variées», que «[q]uelques-unes de [m]es nouvelles appartiennent plus au genre de prose poétique qu'au récit proprement dit» $(\mathrm{ABV})$, il précise cependant que «[d]ans mon cas, la nouvelle tient plus du récit que de l'imaginaire comme tel» (SHSB 0091/1445/5).

Il y a si peu d'écart entre l'auteur et le narrateur, particulièrement dans les nouvelles au JE, comme dans «Un homme de trente ans» (d'ailleurs défini comme «récit», non pas «nouvelle»), qu'il faut se pencher sur ces textes comme des «tranches de vie» de RV/GD/GV.

Le nom de Rossel Vien, l'essayiste, est bien plus proche de son état civil (Russel Vien) que le sont les autres pseudonymes. La légère modification d'une voyelle, $\mathrm{O}$ pour $\mathrm{U}$, constitue à la fois une distanciation et une affirmation. Il se distingue, 
légèrement, de ses antécédents problématiques ${ }^{31}$, pour affirmer une identité d'auteur. Cette première identité est liée à l'histoire, à la recherche, aux faits attestés ${ }^{32}$; les autres identités sont affaire de cœur, d'intimité, toute aussi vraie, mais d'un autre ordre : «Le cœur a ses raisons que la raison ne connaît point» affirme Pascal.

L'invention d'un anonymat via le pseudonyme révèle plus qu'il ne cache, car il laisse entendre une vérité que les faits historiques ne peuvent dévoiler. L'invention est ici matière fondamentale, distinguant le réel de la soi-disant réalité:

La nouvelle est le moule (au sens large), le genre qui me vientnaturellement pour traduireuneexpérience, dessiner un portrait, fixer quelque chose à même le mouvement et le fugitif... Puisque le réel est changement, comme on l'a appris dans les rudiments de la philosophie; le fleuve de Démocrite [sic pour Héraclite] (SHSB 0091/1445/5)!

James Joyce a fort bien capté cette différence: «Mais pour de tels esprits un portrait n'est pas un papier d'identité, mais bien plutôt la courbe d'une émotion.» (Joyce, 1982, p. 313). Lire la fiction de Delaunière/Valais, c'est lire RV.

La fiction, c'est simplement le codage, le camouflage (pour raisons variées), comme il est suggéré dans le Prologue d'«Un homme de trente ans» : «Les noms des personnes et des lieux ont été changés ou chiffrés là où c'était nécessaire.»

Le génie de RV aura peut-être été, en cachant son écriture sous des pseudonymes, de permettre à Russel Vien de faire voir à quel point Rossel était un excellent essayiste, à quel point les Gilles étaient de grands auteurs de nouvelles.

RV s'est toujours senti partagé : «À partir de ce temps je me reconnais mieux tel que je suis aujourd'hui: sauvage, avec un besoin forcené de rire et de danser; sensuel, et vivant toujours dans l'inconfort et le malaise; inquiet, et passant pour bohême [sic]; égoïste, mais refusant une vie réservée à moi-même.» (Delaunière, 1960, p. 195). Par sa fiction, il est entré dans sa division interne.

Il se révèle dans les traces de sa fuite : «Ils [les personnages]se fuient eux-mêmes», «Le jeune novice se fuit», «Le personnage principal de Et fuir encore court partout sans 
trouver le repos» (Blais, 1972, p. 14). «Son angoisse, sa solitude, son masochisme lui donnent l'horreur non seulement de la violence physique, mais de tout ce qui est physiologique. Il veut fuir, s'évader» (Paré, 1960, p. 42).

\section{Virgulation}

Ce côté fuyant ${ }^{33}$, fuyant dans la division même de son être, symboliquement représenté par la variété des pseudonymes, éventuellement assumé comme naturel («le réel est changement» dit l'auteur), devient le signe même de son écriture («une leçon d'écriture» affirme Réginald Martel), transparaissant dans ce que j'appellerais la «virgulation» (ponctuation à virgules) de ses textes, que RV qualifiait de "phrases spaghetti» ${ }^{34}$. La grande majorité des nouvelles de RV, surtout celles qui ne sont pas des récits, est constituée de longs paragraphes ponctués uniquement par des virgules ${ }^{35}$. Paragraphes qui se terminent souvent par des points de suspension. «C'est en fin de compte, écrit l'auteur, une 'tranche' de vécu, prise au cœur et dans la moelle, une tranche découpée en laissant de côté les apprêts et les croûtes.» (SHSB 0091/1445/5) La critique n'a pas toujours apprécié : «Le style très soutenu du texte, écrit dans un français impeccable colle bien au ton urgent du texte, au désespoir qu'il exprime mais il devient, à la longue, agaçant, puisqu'il est caractérisé par l'emploi de longues phrases, couvrant parfois toute une page.» (Marchildon, 1986-1987, p. 53)

Cette physicalité de l'écriture de RV est à l'image de l'homme. La virgule n'est jamais une clôture, elle propulse sans arrêt par devant, elle dénote un équilibre instable dans l'eau héraclitéenne de la vie. Cette ponctuation est le souffle même de l'auteur.

RV n'est certes pas le premier à utiliser cette écriture ouverte. On n'en reviendra pas de la longue continuation de la phrase chez Proust; mais dans l'étalement moderne évident de la forme, on peut songer tout de go à divers auteurs du Nouveau Roman, dont Claude Simon dans Le Palace (1962), par exemple. L'auteure québécoise Marie-Claire Blais s'y adonnera plus tard, surtout dans son cycle Soifs. Ainsi, tout comme Rossel Vien peut être tenu comme un précurseur dans l'écriture à thématique homosexuelle $^{36}$, il peut être tenu comme un précurseur d'une 
certaine modernité d'écriture au Canada, puisque le premier exemple de la virgulation chez lui apparaît dès 1970.

«[L]e style, disait Buffon, est l'homme même» (Buffon, 1894, p. 43). George Sand d'ajouter : «On a dit ' le style, c'est l'homme'. La ponctuation est encore plus l'homme que le style» (Sand, 1873, p. 92).

Ainsi, c'est l'identité d'auteur de RV, multiple, qui survit. Identification avec la face cachée, aussi trouble soit-elle, adéquation entre la fuite et le dire du fuyant, rythmique d'un homme dont le souffle de vie, aussi masqué ou assumé soit-il, se prolonge dans l'écriture et s'éternise dans l'œuvre.

\section{NOTES}

1. Voir Raymond Hébert, ci-dedans.

2. Archives de Bernard Vien, confiées à Raymond Hébert en 2018 (non traitées), ci-après ABV. Nous remercions Raymond Hébert de nous avoir permis de les consulter.

3. ABV. Si l'utilisation de Russell avec deux L par Bernard Vien peut être considéré un lapsus - car Bernard Vien utilisera la graphie du certificat de naissance (Russel avec un seul L) ailleurs dans le document - ce n'est certes pas le cas de la modification qu'apporte lui-même l'auteur adolescent.

Curieusement une fiche des Clercs de Saint-Viateur (ABV) chez qui Rossel Vien fit ses études et prit l'habit l'identifie comme VIEN, Russell-Joseph-Paul-Émile.

Ailleurs, dans ses notes à l'intention de Raymond Hébert, Bernard Vien explique la présence du nom Russell dans la région de Roberval/Lac-Saint-Jean par la présence d'aventuriers écossais et irlandais parmi les populations amérindiennes, de sorte que la graphie anglophone Russell se retrouve même chez des familles canadiennes-françaises. Il signale ceux de Russell Bolduc, Russell Boivin, Russell Dion.

4. On notera que Placide Gaboury du Collège de Saint-Boniface obtient le $3^{\mathrm{e}}$ prix.

5. Une recherche entreprise pour moi par Bernard Mulaire, que nous remercions, auprès du Directeur de l'état civil du Québec laisse entendre que si le nom inscrit à l'acte de baptême de Rossel est indiqué dans le passeport de 1972, soit Russel, c'est que jusqu'au début des années 1980, quand le Code civil du Québec a été modifié, entre autres au sujet de la loi sur la famille, pour permettre aux femmes d'obtenir une identité légale autre que celle de leur mari, on pouvait changer son nom par acte notarié dit Deed 
Poll commun aux pays du Commonwealth. À la demande d'un passeport, on devait alors présenter son acte de baptême et le Deed Poll pour utiliser son nouveau nom, mais le passeport citait l'acte de baptême.

Une attestation que j'ai reçue du Directeur de l'état civil au Québec confirme que le nom de naissance est Russel et qu'aucun changement de nom n'a été consigné au registre de l'état civil.

Quoiqu'il en soit, notre auteur aura réussi à rendre officieusement officiel le nom Rossel comme le démontrent plusieurs cartes d'identité (ABV): Union des Artistes, Services de santé du Manitoba, possiblement le Numéro d'assurance sociale (le nom sur le facsimilé est illisible, mais la carte est signée Rossel Vien), l'auteur signe Rossel Vien sur son certificat de statut indien émis au nom de Russel, enfin il recevait correspondance, avis, chèques au nom de Rossel Vien.

6. C'est une nouvelle que RV signe sous le nom de Rossel Vien. Puisqu'elle lui a été demandée pour un numéro spécial de la revue sur la littérature franco-manitobaine, il doit donc s'identifier.

À une demande de correction du texte avant publication, portant sur l'identification «Déné» qui semble une anomalie, il répond : «Je suis Montagnais, mais à North Battleford, je suis Déné, que voulez-vous.» (Fonds Rossel Vien, Centre du Patrimoine, Société historique de Saint-Boniface, 0091/1445/25)

7. Si le médecin qui a pratiqué l'autopsie était anglophone, il ne serait pas étonnant qu'il ait inconsciemment inscrit la graphie usuelle en langue anglaise. Quoiqu'il en soit, il est curieux de constater que jusqu'à la fin l'identité de RV semble échapper.

8. Il signera du nom de Rossel Vien le poème «Dénuement» dans Le Devoir, samedi 16 novembre 1957, p. 24. On peut lire dans une note qu'il m'a adressé lorsque je préparais l'Anthologie de la poésie franco-manitobaine : «R. Vien : tous les poèmes envoyés au Devoir l'ont été aux environs de 1960. J'en ai retrouvé deux autres qui me le confirment. Donc, indiquer : circa 60, faute de mieux. Je n'ai pas noté les dates. Et je me demande pourquoi j'envoyais cela à ce journal. On manquait de revues...?» (SHSB 0091/1446/26) À part «Dénuement», nous n'avons pas encore pu retracer les poèmes signalés par RV envoyés au Devoir. Il se peut que RV les ait envoyés, mais qu'ils n'aient pas été publiés.

9. Voir Raymond Hébert, Armelle St-Martin, ci-dedans.

10. Gaston Bellemare, président-fondateur du Festival international de la poésie de Trois-Rivières, m’a indiqué qu'il s'agit de Gérald Godin.

11. Comité composé alors de : Gérard Pelletier, Pierre Elliott Trudeau, du journaliste Jean-Louis Gagnon, des auteurs Robert Élie et Marcel Dubé, ainsi que de Gilles Marcotte. 
12. Cette «familiarité» n'a pas empêché Marcotte, qui a louangé Gilles Delaunière pour Un homme de trente ans, de critiquer le Journal sans suite de Rossel Vien : «qui doit apprendre que le récit du voyage ne vit pas seulement de la variété des décors, mais aussi, et surtout, d'affirmation personnelle et de style.» (Marcotte, 1965, p. 6).

13. On note dans les archives de l'auteur, Fonds Rossel Vien, à la Société historique de Saint-Boniface que la correspondance maintenue avec Gilles Delaunière par divers individus aux Écrits du Canada français et chez Hurtubise HMH est adressée à Rossel Vien.

14. Voir ci-dedans.

15. Une analyse de Jacques Michon en 2007 confirme cette opinion: les fondateurs des maisons ont quitté ou se sont fait mettre à la porte, les défis sont nombreux, il y a des déficits, notamment chez Hurtubise HMH, Fides et aux Éditions du Jour (Michon, 2007, p. 218).

16. Toutes les lettres référencées dans cette section se trouvent aux archives de la Société historique de Saint-Boniface, Fonds Rossel Vien, dossier Correspondance Hurtubise (SHSB 0091/1445/12).

17. Un court extrait est publié en 1999 sous le nom de Rossel Vien dans le volume $B L E ́$, fêtant les 25 ans d'existence des Éditions du Blé (Léveillé, 1999, p. 116-117).

Puisque cette publication fut produite sept ans après le décès de l'auteur, il me semble que le texte a dû m'être remis au moment où je préparais, avec Rosmarin Heidenreich et Josée Vaillancourt, l'édition du numéro spécial de la revue Prairie Fire en 1990 sur la littérature franco-manitobaine. C'est «Retour à Saskatoon» qui sera retenu pour publication. Il est clair toutefois que RV savait que le texte serait publié sous le nom Rossel Vien, même si le manuscrit donne Gilles Delaunière comme auteur. Le titre original retenu puis rayé par Vien était «Le désir».

18. À preuve, la notice pour Gilles Delaunière, qui précède la publication des deux nouvelles, «La coulée» et "Les roses de papier» dans le $\mathrm{n}^{\circ} 43$ des Écrits $d u$ Canada français, laisse entendre: «À paraître prochainement, un second recueil de nouvelles.» $\mathrm{Ce}$ sera Les deux frères de Gilles Valais.

19 Voir ci-dedans.

20. «Je ne savais absolument rien de Rossel quand j'ai voulu l'inclure dans le Répertoire et je lui ai demandé de la rédiger lui-même, mais il préférait que je la signe. Je n'ai pas porté attention à sa publication de nouvelles sous des pseudonymes.» [Courriel de A. Saint-Pierre à J.R. Léveillé, 9 juillet 2019 - archives de J.R. Léveillé]

21. Au désespoir de son éditrice qui ne pouvait pas organiser de lancement publique ni de battage publicitaire : «Rossel m’a apporté 
son manuscrit Les deux frères. J'ai accepté ce nom de plume sans dire un mot, mais après j'ai insisté pour son véritable nom pour Les deux soeurs et il a refusé de nouveau.» [Courriel de A. Saint-Pierre à J.R. Léveillé, 8 juillet 2019, archives de J.R. Léveillé]

22. La liste que m’a remise RV, en 1988, identifie les poèmes préalablement publiés : "Mon peuple» et « Nuit» dans Le Devoir, «Insolente durée» dans le Courrier de Saint-Boniface et «Le bison exterminé» dans les Cahiers franco-canadiens de l'Ouest. Cette liste est divisée en deux sections : Poèmes de détachement (9 poèmes) et Poèmes du pays ( 3 poèmes). Un poème "Chambre à louer», publié dans le Courrier est rayé de la liste. Nous n'avons pas encore repéré dans les archives du Devoir, les poèmes identifiés par RV, par contre nous avons trouvé un poème, «Dénuement», signé Rossel Vien, publié le samedi 16 novembre 1957, à la page 24. Il est possible que le titre ait été modifié ou qu'il s'agisse d'un tout autre poème. Après tout, si les poèmes de cette liste fournie par Rossel Vien semblent constituer la majeure partie de sa production, ils n'en constituent pas la totalité. J'avais repéré ou eu accès à d'autres poésies qui ont été publiées dans l'Anthologie de la poésie franco-manitobaine.

23. Cf. supra, note 7.

24. Mes remerciements au frère Wilfrid Bernier, archiviste des C.SV., qui m’a toutefois précisé que les listes des élèves du Collège Notre-Dame de Roberval sont incomplètes. L'archiviste m'a rappelé qu'en venant étudier au Séminaire de Joliette en 1944, RV se trouvait alors dans la grande région dite de Lanaudière, ce qui a pu influencer le choix du nom du pseudonyme, et fourvoyer certains des premiers commentateurs de RV.

25. Il fait de même en 1981, en écrivant à Victor-Lévy Beaulieu : «G. Delaunière est un pseudonyme déjà utilisé pour d'autres nouvelles.» (SHSB 0091/1445/12)

26. À René Godenne, spécialiste français de la nouvelle, il écrit: «Vous savez sans doute que Gilles Delaunière est un pseudonyme. J'en ai adopté un autre; je trouvais nécessaire de me dissocier de certaines attitudes exprimées dans mon premier texte littéraire que les Écrits du Canada français ont publié de moi [...] Je vous ai expédié dernièrement un exemplaire de ce deuxième recueil signé Gilles Valais. La dernière nouvelle que j'ai publiée («La statue») a paru dans la revue Les Moissons [...]» (SHSB 0091/145/6). «La statue» a été publiée sous le nom de Rossel Vien

27. Notons les nouvelles suivantes de Gilles Delaunière présentées à Hurtubise HMH en 1979 et aux Quinze en 1980 : «La statue» publiée sous Rossel Vien en 1982, «Le monastère» publiée sous Gilles Valais en 1986, «Lettre de Maud» publiée sous Gilles Valais en 1985; présentée à Hurtubise HMH en 1979 : «Les deux frères» 
publiée sous Gilles Valais en 1982; présentée aux Écrits du Canada français en 1979: «Chibougamau» publiée sous Gilles Valais en 1982; présentée aux Quinze en 1980 : «Le fils unique» publiée sous Gilles Valais en 1990; présentée aux Écrits du Canada français en 1985 : «Quantièmes», extrait publié sous Rossel Vien en 1999.

28. Cf. supra, note 17.

29. Voir ci-dedans.

30. L'ébauche des propos de RV sur le genre de la nouvelle contenu dans ce fonds relève sans doute d'une correspondance entretenue avec René Godenne (note 26 ci-dessus) datée du 14 décembre 1983 : «Cher monsieur, En réponse à votre demande datée du 28 octobre, que l'éditeur HMH de Montréal m'a fait parvenir, j'ai griffonné quelques réflexions sur la nouvelle. Je n'ai pas de théorie sur la nouvelle. Je pourrais peut-être en élaborer une si je m'y arrêtais.»

31. Voir ci-dedans Lise Gaboury-Diallo.

32. RV, lui-même, en fait la distinction en s'expliquant sur le genre de la nouvelle, mettant cette distinction entre parenthèses : «(J'ai touché aussi au journalisme et à l'histoire.)» (SHSB 0091/1445/5)

33. On pourra noter dans les Archives de Bernard Vien et dans le Fonds Rossel Vien de la Société historique de Saint-Boniface que ce "gommage» transparaît dans une variété d'ébauches de biobibliographies que prépare l'auteur. Certes, ces brouillons peuvent s'adresser à diverses fins. Il demeure curieux toutefois de voir que dans la notice présentant l'auteur du «Journal sans suite» dans les Écrits du Canada français, il est mentionné une œuvre : la traduction d' «un ouvrage sur Louis Riel (Éditions du Jour). Aucune référence à son Histoire de Roberval qui avait pourtant été louangée. Elle est aussi négligée ailleurs au profit de La radio française dans l'Ouest. Dans une bibliographie sommaire, il donne Gilles Valais comme son pseudonyme. Ailleurs il raye une mention : «Un de ses ouvrages a été finaliste pour le prix littéraire France-Québec.»

34. C'est le dramaturge franco-manitobain new-yorkais Guy Gauthier qui me rappelle cette expression: «Tu avais raison de signaler ses longues phrases, qui pourtant ne donnent pas l'impression d'un relâchement, ou d'un stream-of-consciousness. Il les nommait, dans notre correspondance, "mes phrases spaghetti".» (Correspondance personnelle Guy Gauthier/J.R. Léveillé, 26 mai 2020.). Gauthier et Vien se sont connus à Winnipeg, sont demeurés amis et correspondants.

35. Les trois premières œuvres (un récit, une nouvelle et un journal) utilisent une forme standard. «Les aveugles de Matamoros» en 1970 est sa première fiction à utiliser la virgulation. Six des sept nouvelles de Et fuir encore sont constitués d'une écriture à virgules. De ces nouvelles, seule «Oriel» présente une forme standard; sa 
composition pré-date possiblement «Les aveugles de Matamoros» qu'elle précède dans la série du recueil. Par la suite, RV poursuivra cette forme à virgules, ou mélangera à sa virgulation dans un même texte des formes plus standard («Les deux sœurs», «Lettre de Maud», «Le monastère»), réservant la forme plus conventionnelle au roman ou au récit.

36. Voir Bernard Mulaire ci-dedans.

\section{BIBLIOGRAPHIE}

ANONYME (1947) «Lauréats du Xème Concours de Vacances», Le Devoir, vendredi 21 novembre, p. 5)

ANONYME (1960) «Les écrivains», La Presse, édition provinciale, 19 novembre 1960, p. 28.

A.S.P. [SAINT-PIERRE, Annette] (1984) «Rossel Vien», Répertoire littéraire de l'ouest canadien, Saint-Boniface, Centre d'études franco-canadiennes de l'Ouest, p. 355-358.

BEAULIEU, Victor-Lévy (1972a) «Écrire, selon Lévy», Le Devoir, samedi 11 mars, p. 14.

(1972b) «L'enjeu de Jacques Ferron, être plus grand que le pays», Le Devoir, samedi 9 décembre, p. 19.

(1987) dans Dictionnaire des œuvres littéraires du Québec, Tome V : 1970-1975 (sous la direction de Maurice Lemire), Montréal, Fides, 1133 p.

BLAIS, Jean-Éthier (1964) «Les Écrits du Canada français numéro 18. Du meilleur et du pire.», Le Devoir, 17 octobre, p. 15.

(1972) «Delaunière et Maillet. Les amours abusives.», Le Devoir, 12 février, p. 14.)

BOIVIN, Aurélien et BOURGEOIS, Jean-Marc (1986) «Vien, Russel» dans Littérature du Saguenay-Lac-Saint-Jean : répertoire des oeuvres et des auteurs, $2^{\mathrm{e}}$ édition revue et mise à jour, Alma, Éditions du Royaume, 242 p.

BUFFON (1894) Discours sur le style, $5^{\mathrm{e}}$ édition, Paris, Librairie Ch. Delagrave, $48 \mathrm{p}$.

DELAUNIÈRE, Gilles (1960), «Un homme de trente ans», Écrits du Canada français, $\mathrm{n}^{\circ} 6$, p. 155-211.

DUHAMEL, Roger (1972) «Deux écrivains nous sont nés», Le Droit, samedi 26 février, p. 13.

ÉCRITS DU CANADA FRANÇAIS (1960) «Delaunière, Gilles, 'Un homme de trente ans'», $\mathrm{n}^{\circ} 6, \mathrm{p} .156$. 
G.G. [GODIN, Gérald] (1960), «Écrits du Canada Français - Une littérature vivante», Le Nouvelliste, Trois-Rivières, samedi 12 mars, p.14.

G.H. (1961) «Les Écrits du Canada français. Lieu de rencontre de diverses tendances littéraires», Le Devoir, 28 janvier, p. 10

GUÉRIN, M.-A. et HAMEL, Réginald (2001) Dictionnaire des poètes d'ici, de 1606 à nos jours, Montréal, Guérin éditeur, 1957 p.

(2005) Dictionnaire Guérin des poètes d'ici, de 1606 à nos jours, Montréal, Guérin éditeur, 1359 p. [v. p. 1316].

HAMEL, Réginald, HARE, John et WYCZYNSKI, Paul, dir. (1989) Dictionnaire des auteurs de langue française en Amérique du Nord, Montréal, Fides, 1364 p.

JOYCE, James (1982) Portrait de l'artiste en jeune homme, Paris, Gallimard, La Pléiade, tome I, 2096 p.

LÉVEILLÉ, J.R. (1990), Anthologie de la poésie franco-manitobaine, SaintBoniface, Éditions du Blé, 592 p.

LÉVEILLÉ, J.R. (dir.) (1999) BLÉ, Saint-Boniface, Éditions du Blé, 1999, 205 p.

LÉVEILLÉ, J.R. (2020) «Rossel Vien», préface dans Vien, Rossel, Et fuir encore, Saint-Boniface, Éditions du Blé, p. 8.

MARCHILDON, Daniel (1986-1987) «'Les deux soeurs': Deux textes pénétrant de jeunesse», Liaison, n 41 , p.52-53.

MARCOTTE, Gilles (1960) «Les Écrits du Canada français - 6e (Asselin, Delaunière, Straram)», Le Devoir, 19 mars, p. 11.

(1965) «La réalité, l'imagination, les écrivains...» La Presse, samedi 30 janvier, Supplément, p. 6.

MARTEL, Réginald (1972) «En pièces détachées», Le Devoir, samedi 12 février, p. C3.

MICHON, Jacques (2007) «L'édition en français», dans GERSON, Carole et MICHON, Jacques (dir.), Histoire du livre et de l'imprimé au Canada, (vol. III : de 1918 à 1980), Montréal, PUM, 672 p.

PARÉ, Jean (1960) «La vie littéraire», La Presse, samedi 12 mars, p. 42.

SAND, George (1873) Impressions et souvenirs, Paris, Michel Lévy Frères, $366 \mathrm{p}$.

SOCIÉTÉ HISTORIQUE DE SAINT-BONIFACE, Fonds Rossel Vien, 0091/1445/5.

Fonds Rossel Vien, 0091/1446/26. 
Fonds Rossel Vien, 0091/1445/12.

THÉRIO, Adrien, dir. (1972) Livres et auteurs québécois 1972, Montréal, Éditions Jumonville, 1972, 444 p.

TOUSSAINT, Ismène (2000) «Rossel Vien», Encyclopédie canadienne, Montréal, Stanké, 2642 p.

VIEN, Rossel (1962) «Voyage sans suite», Écrits du Canada français, $\mathrm{n}^{\mathrm{o}} 18$, p. 147-219.

_ (1990) «Retour à Saskatoon», Prairie Fire, vol. 11, n 1, p. 130-136 